\title{
Classification of PR-positive and PR- negative subtypes in ER-positive and HER2- negative breast cancers based on pathway scores
}

Taobo Hu', Yan Chen ${ }^{2}$, Yiqiang Liư ${ }^{3}$ Danhua Zhang ${ }^{4}$, Jiankang Pan ${ }^{5^{*}}$ and Mengping Long ${ }^{3^{*}}$

\begin{abstract}
Purpose: PR loss in ER+/HER2- breast cancer indicates worse prognosis and insensitivity to anti-estrogen therapy, while the mechanisms of PR loss in ER+/HER2- breast cancer remain unrevealed.

Methods: In this study, ER+/PR+/HER2- and ER+/PR-/HER2- breast cancer cases from TCGA were used. 1387 pathways were analyzed and used as variables for classifying the two groups with LASSO regression.

Results: ER+/PR+/HER2- and ER+/PR-/HER2- breast cancer groups can be classified by a combination of 13 pathways using their activity score. Among the 13 pathways, those involving growth factors and ion-channel transporters were most significant in the distinction, followed by pathways involving immune modulation and cell metabolism. Two growth factor pathways, EGF and IGF-1, were deferentially regulated in ER+/PR+/HER2- and ER+/ PR-/HER2- groups.
\end{abstract}

Conclusions: In conclusion, this study indicated in ER+/HER2- breast cancers the various status of PR expression can be an indication of molecular variation, particularly for the growth factor pathway activation.

Keywords: Estrogen receptor, Progesterone receptor, Pathway activities, LASSO, Growth factor

\section{Introduction}

About $80 \%$ of breast cancers were hormonal receptorpositive which means they express at least one of the two hormonal receptors, estrogen receptor (ER) and progesterone receptor (PR) $[1,2]$. Both ER and PR are ligand-activated transcription factors that promote the expression of specific gene sets by binding to their promoters [3]. Although the expression of ER and PR were often closely correlated and highly consistent, there is still discordance in some breast cancers. It was reported

\footnotetext{
*Correspondence: 188202109@csu.edu.cn; mplong@connect.ust.hk

${ }^{5}$ Department of Orthopedics, The Second Xiangya Hospital, Central South University, Changsha, Hunan, China

${ }^{3}$ Department of Pathology, Peking University Cancer Hospital, Beijing, China Full list of author information is available at the end of the article
}

that $15 \%$ of ER-positive breast cancer were PR negative while in PR-positive breast cancer, only $2 \%$ were ERnegative [4], which suggests that ER expressed more widely than PR. Indeed, about $12 \%$ of all breast cancer patients have the hormonal receptor status as ER+/PR[4]. One of the possible mechanisms for PR loss could be the copy number loss of the PGR gene which encodes for PR.

The expression of PR was mostly controlled by activated ER [5], while it can also be regulated by growth factor pathways [6] and cyclin D1 [7]. Analysis of both the Surveillance, Epidemiology and End Results (SEER) [4] and the National Cancer Database (NCD) [8] datasets has confirmed that ER+/PR- breast cancer has worse survival than $\mathrm{ER}+/ \mathrm{PR}+$ breast cancers. Moreover, it was

(c) The Author(s). 2021 Open Access This article is licensed under a Creative Commons Attribution 4.0 International License, which permits use, sharing, adaptation, distribution and reproduction in any medium or format, as long as you give appropriate credit to the original author(s) and the source, provide a link to the Creative Commons licence, and indicate if changes were made. The images or other third party material in this article are included in the article's Creative Commons licence, unless indicated otherwise in a credit line to the material. If material is not included in the article's Creative Commons licence and your intended use is not permitted by statutory regulation or exceeds the permitted use, you will need to obtain permission directly from the copyright holder. To view a copy of this licence, visit http://creativecommons.org/licenses/by/4.0/. The Creative Commons Public Domain Dedication waiver (http://creativecommons.org/publicdomain/zero/1.0/) applies to the data made available in this article, unless otherwise stated in a credit line to the data. 
shown that the ER+/PR- group was more resistant to selective estrogen receptor modulator (SERM) therapies $[9,10]$. The exact mechanism of the association between PR loss and worse prognosis was remained to be elucidated although several clues existed [11]. One possible mechanism was that the relative overexpression of HER2 in the $\mathrm{ER}+/ \mathrm{PR}$ - group compared with the $\mathrm{ER}+/ \mathrm{PR}+$ group made $\mathrm{ER}+/ \mathrm{PR}$ - breast cancer resistant to tamoxifen $[12,13]$. However, in ER+/HER2- breast cancer, the effect of PR expression on prognosis still exists, indicating the presence of other mechanisms [14]. PR has long been considered as the downstream gene of ER since its expression was shown to be induced by estradiol [15], while recent researches proved that PR has strong negative regulatory effect on ER activity [16]. The complex correlation between ER and PR makes it essential to elucidate the molecular characteristiscs and clinical significance of PR loss in ER+/HER2- breast cancer [16].

In this study, we analyzed and compared the pathway activities in ER+/PR+/HER2- and ER+/PR-/HER2- breast cancer using transcriptomic and gene amplification data from TCGA. The two groups were abbreviated as ER+/ $\mathrm{PR}+$ and ER+/PR-, thus all the studied patients were HER2- unless otherwise specified.

\section{Results}

\section{Clinicopathological characteristics and survival analysis of} the ER+/PR + and ER+/PR- breast cancer patients

In the TCGA cohort, 592 patients have information on ER and PR expression based on immunohistochemical staining. The expression of ER and PR were reported as the percentage of cells with positive expression. ER + and $\mathrm{PR}+$ were defined as expressions of more than $1 \%$, according to the ASCO/CAP guideline [17].

Cases with positive expression of ER and PR were further subgrouped into 10 categories with a $10 \%$ interval. By this definition, 123 patients belong to the ER-/PR-, while 60 cases were ER+/PR- and 409 cases were ER+/ $\mathrm{PR}+$ (Fig. 1).

Clinicopathological characteristics of the ER+/PR- and $\mathrm{ER}+/ \mathrm{PR}+$ groups were compared (Table 1$)$. In concordance with previous studies, the two groups showed a significant difference in clinicopathological characteristics including clinical stage and nodal status. Specifically, $\mathrm{ER}+/ \mathrm{PR}$ - group patients have both more advanced clinical stage and nodal status. In terms of PAM50 intrinsic subtypes distribution of the two groups, it was found that the PR-group was more enriched in luminal B and basal-like type than the PR + group. In addition, comprehensive molecular portraits of the two groups were analyzed including DNA methylation, copy number variation, and miRNA profile which have all been tested and clustered in the previous study [18]. Distributions in methylation cluster and copy number cluster were found

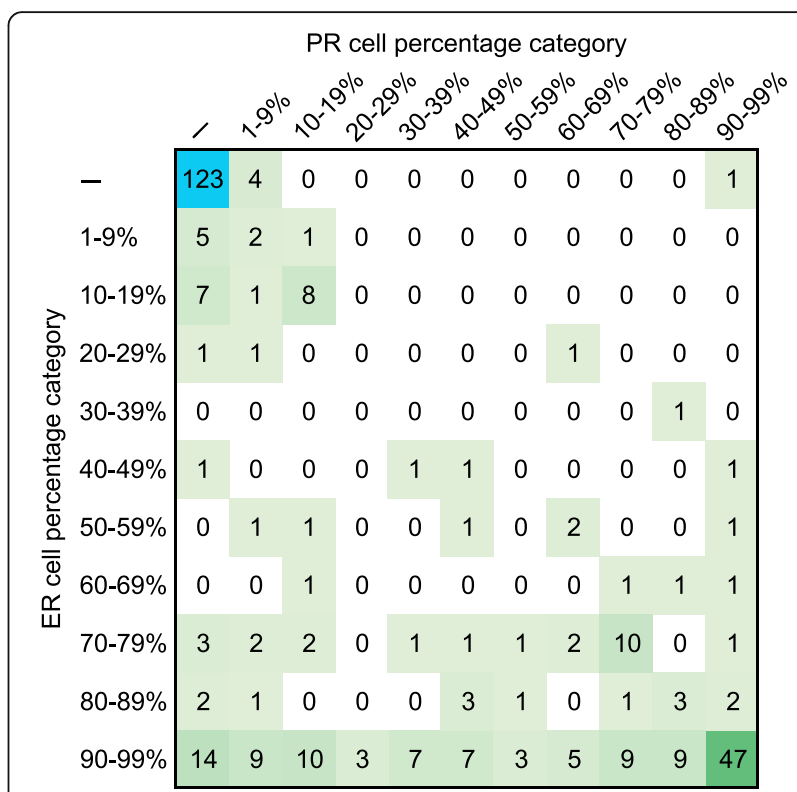

Fig. 1 Cell percentage categories of ER and PR expression in HER2breast cancers from TCGA. The case number of each specific ER and PR expression status was summarized and presented. ER and PR expression were reported either as negative or as the percentage of cells showed positive expression which was subgrouped into 10 categories with a $10 \%$ interval

to be different between the two groups while no difference was detected in the miRNA cluster distribution. For the DNA methylation cluster, the PR- group was enriched in cluster 5 which was a cluster that overlapped with basal-like mRNA subtype while the PR + group was more enriched in cluster 2 which seems to be a mixture of mRNA subtypes. For copy number cluster, PR- group was enriched in cluster 2 with PR + enriched in cluster 1 . Copy number clusters 1 and 2 were previously found to be correlated with luminal A and basal-like subtypes respectively. All the above results indicated that molecularly PR-group was more similar with basal-like subtype and has a more advanced clinical stage than the PR+ group.

The survival analyses were performed by comparing the overall survival of the ER+/PR-/HER2- and ER+/ $\mathrm{PR}+/$ HER2- group (Fig. 2). In accordance with previous studies, the overall survival of ER+/PR-/HER2- was significantly poorer than the ER+/PR+/HER2- group. To further validate the result, the same analysis was performed in the Surveillance, Epidemiology, and End Results (SEER) database (Fig. 2). A total of 110,930 ER+/ HER2- breast cancer patients were included in the SEER dataset analysis with 97,397 of them being PR + and 13, 533 of them being PR-. The clinicopathological characteristics of the included population were presented in Table S1. Li et al. have previously studied the ER+/PR + 
Table 1 Patient Clinicopathological features of the two selected groups and the multivariate and univariate analysis in the TCGA dataset

\begin{tabular}{|c|c|c|c|}
\hline Characteristics & ER+/PR-/HER2- & ER+/PR+/HER2- & $p$ \\
\hline \multicolumn{4}{|l|}{ Age } \\
\hline$<35$ & $2(3.3)$ & $11(2.7)$ & 0.418 \\
\hline $35-49$ & $10(16.7)$ & $97(23.7)$ & \\
\hline $50-69$ & $31(51.7)$ & $218(53.3)$ & \\
\hline $70+$ & $17(28.3)$ & $83(20.3)$ & \\
\hline AJCC Stage ${ }^{a}$ & & & 0.005 \\
\hline Stage I & $9(15.0)$ & $83(20.9)$ & \\
\hline Stage $\|$ & $27(45.0)$ & $222(55.8)$ & \\
\hline Stage III & $19(31.7)$ & $86(21.6)$ & \\
\hline Stage IV & $5(8.3)$ & $7(1.8)$ & \\
\hline Histology & & & 0.667 \\
\hline Infiltrating Ductal Carcinoma & $46(76.7)$ & $316(77.3)$ & \\
\hline Infiltrating Lobular Carcinoma & $6(10.0)$ & $54(13.2)$ & \\
\hline Mixed Histology & $4(6.7)$ & $16(3.9)$ & \\
\hline Others & $4(6.7)$ & $23(5.6)$ & \\
\hline PAM50 & & & $<0.001$ \\
\hline Luminal A & $12(27.9)$ & $181(65.8)$ & \\
\hline Luminal B & $18(41.9)$ & $81(29.5)$ & \\
\hline Basal-like & $7(16.3)$ & $3(1.1)$ & \\
\hline HER2-enriched & $4(9.3)$ & $6(2.2)$ & \\
\hline Normal-like & $2(4.7)$ & $4(1.5)$ & \\
\hline Methylation Cluster ${ }^{b}$ & & & $<0.001$ \\
\hline Cluster 1 & $8(13.3)$ & $80(19.6)$ & \\
\hline Cluster 2 & $9(15.0)$ & $124(30.3)$ & \\
\hline Cluster 3 & $9(15.0)$ & $40(9.8)$ & \\
\hline Cluster 4 & 19 (31.7) & 149 (36.4) & \\
\hline Cluster 5 & $12(20.0)$ & $16(3.9)$ & \\
\hline NA & $3(5.0)$ & $0(0.0)$ & \\
\hline CN Cluster ${ }^{\mathrm{b}}$ & & & 0.001 \\
\hline Cluster 1 & $17(30.4)$ & $42(10.4)$ & \\
\hline Cluster 2 & $13(23.2)$ & $161(40.0)$ & \\
\hline Cluster 3 & 11 (19.6) & 73 (18.2) & \\
\hline Cluster 4 & $9(16.1)$ & $71(17.7)$ & \\
\hline Cluster 5 & $6(10.7)$ & 55 (13.7) & \\
\hline miRNA Cluster ${ }^{b}$ & & & 0.788 \\
\hline Cluster 1 & $4(7.8)$ & $29(7.8)$ & \\
\hline Cluster 2 & $8(15.7)$ & 70 (18.8) & \\
\hline Cluster 3 & $4(7.8)$ & $18(4.8)$ & \\
\hline Cluster 4 & $13(25.5)$ & $107(28.7)$ & \\
\hline Cluster 5 & $7(13.7)$ & $33(8.8)$ & \\
\hline Cluster 6 & $9(17.6)$ & $83(22.3)$ & \\
\hline Cluster 7 & $6(11.8)$ & $33(8.8)$ & \\
\hline
\end{tabular}


A

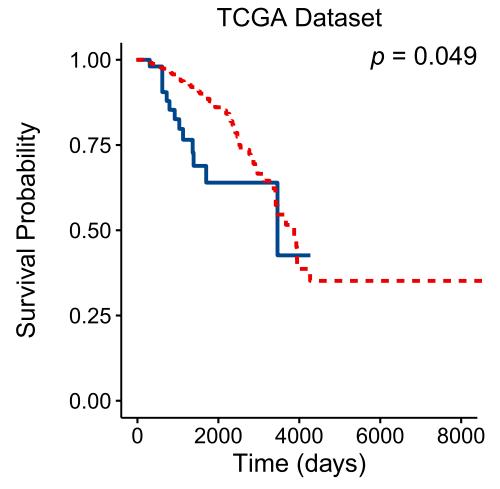

Number at risk

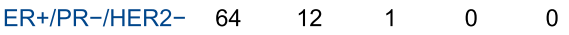

ER+/PR+/HER2- $422 \quad 96 \quad 12 \quad 5 \quad 2$
B

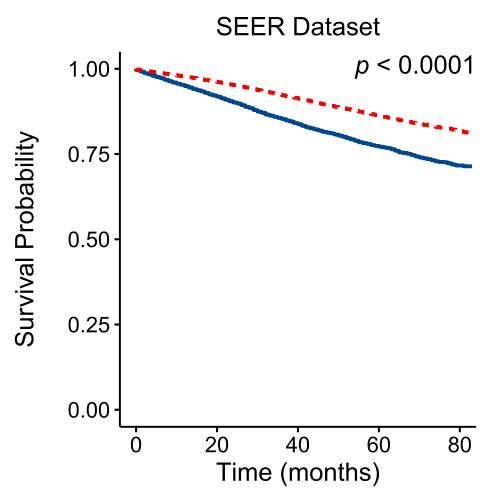

Number at risk

Fig. 2 Survival analysis of the ER+/PR + and ER+/PR- groups. Kaplan-Meier survival curves were plotted and the log-rank test was performed to compare the overall survival of ER+/PR + and ER+/PR- groups in TCGA a and SEER $\mathbf{b}$ database

and ER+/PR- group in the SEER dataset while not considering the status of the HER2 receptor [4].

Both multivariate and univariate analyses were conducted to determine the prognostic value of different clinicopathological features including PR status in all the selected ER+/HER2- patients (Table 2). In univariate analysis, it was found that age, disease stage, tumor size and nodal status were significant prognostic factors for overall survival while factors including age, disease stage, tumor size and copy number cluster were statistically significant in multivariate analysis.

\section{Classification of ER+/PR + and ER+/PR- breast cancer with pathway activities using LASSO methodology}

A strategy named least absolute shrinkage and selection operator (LASSO) was used to select the most accurate and compact set of pathways that can differentiate the $\mathrm{ER}+/ \mathrm{PR}+$ and $\mathrm{ER}+/ \mathrm{PR}$ - group. Logistic-LASSO is a regression method that minimizes the usual sum of squared errors which penalizes the regression coefficients. From the 1387 variables/pathways pool, LASSO was able to reduce the number of pathways needed down to thirteen in the final model (Fig. 3). The final model with all the selected pathways and statistics is shown in Table 3. In the final model, the ER+/PR+/ HER2- and ER+/PR-/HER2- groups can be distinguished by pathway activities of the 13 pathways, achieving a 0.8625 Area Under the receiver operation characteristic Curve (AUC) (Fig. 3). The most distinct pathways between $\mathrm{PR}+$ and $\mathrm{PR}$ - groups were growth factors and ion-channel transporter. Also, the FOXA1 pathway which represents the luminal features, and the NOTCH1 pathway which controls cell proliferation were included in the selected pathways.

\section{EGFR and IGF-1 pathway were deferentially regulated in $\mathrm{ER}+/ \mathrm{PR}+$ and ER+/PR- breast cancer}

In the 13 selected pathways used in the final model, three of them were associated with growth factors including epidermal growth factor receptor (EGFR) and insulin-like growth factor-1 (IGF-1), indicating the direct association between growth factor pathway activation and PR loss. Previous studies have shown that in breast cancer cell lines, activation of EGF or IGF-1 pathways can sharply lower the expression of PR [6]. However, in this study, it was found that the EGFR pathway was more activated in the PR- group while the IGF-1 pathway was more activated in the PR + group (Fig. 4).

The correlation between the pathway score with the mRNA expression value of the $P G R$ gene was plotted (Fig. 5). The IGF-1 pathway was found to be positively correlated with the expression of $P G R$ while the EGFR pathway was negatively correlated, consistent with our above findings. To further look at the impact of the three growth factor pathways on the prognosis, survival analysis was performed between the pathway-defined subtypes by classifying the studied TCGA population into high-activity and low-activity groups according to the value of pathway score (Fig. 6). In the three analyzed pathways, only the IGF1R pathway-defined two groups showed survival difference while the other two pathways did not show a significant difference which was not surprising due to the fact that the IGF1R pathway score showed the greatest difference between $\mathrm{PR}+$ and $\mathrm{PR}$ - group in 
Table 2 Univariate and multivariate analysis of the prognostic value of clinicopathological features in ER+/HER2- patients using Cox proportional hazard modeling in the TCGA dataset

\begin{tabular}{|c|c|c|c|}
\hline Dependent & No. & HR (univariable) & HR (multivariable) \\
\hline \multicolumn{4}{|l|}{ Subtype } \\
\hline ER+/PR-/HER2- & 60 & - & - \\
\hline $\mathrm{ER}+/ \mathrm{PR}+/ \mathrm{HER} 2-$ & 409 & $0.65(0.34-1.25, p=0.196)$ & $0.77(0.21-2.84, p=0.697)$ \\
\hline \multicolumn{4}{|l|}{ Age } \\
\hline $70+$ & 100 & - & - \\
\hline $50-69$ & 249 & $0.60(0.34-1.05, p=0.075)$ & $0.21(0.08-0.57, p=0.002)$ \\
\hline $35-49$ & 107 & $0.26(0.12-0.57, p=0.001)$ & $0.05(0.01-0.22, p<0.001)$ \\
\hline$<35$ & 13 & $1.05(0.31-3.57, p=0.941)$ & $0.00(0.00-\operatorname{Inf}, p=0.999)$ \\
\hline \multicolumn{4}{|l|}{ AJCC Stage } \\
\hline Stage I & 92 & - & - \\
\hline Stage II & 249 & $1.45(0.66-3.18, p=0.354)$ & $8.22(1.55-43.47, p=0.013)$ \\
\hline Stage III & 105 & $1.75(0.74-4.15, p=0.200)$ & $6.55(0.47-92.01, p=0.163)$ \\
\hline Stage IV & 12 & $7.58(2.90-19.79, p<0.001)$ & $133.80(4.74-3775.01, p=0.004)$ \\
\hline \multicolumn{4}{|l|}{ Histology } \\
\hline IDC & 362 & - & - \\
\hline ILC & 60 & $0.69(0.29-1.61, p=0.387)$ & $1.35(0.32-5.71, p=0.682)$ \\
\hline Mixed Histology & 20 & $0.82(0.29-2.27, p=0.700)$ & $2.06(0.39-10.89, p=0.396)$ \\
\hline Others & 27 & $1.29(0.55-3.04, p=0.562)$ & $1.14(0.16-7.96, p=0.893)$ \\
\hline \multicolumn{4}{|l|}{ pT } \\
\hline $\mathrm{T} 1$ & 138 & - & - \\
\hline $\mathrm{T} 2$ & 256 & $1.30(0.70-2.38, p=0.406)$ & $0.27(0.07-1.03, p=0.055)$ \\
\hline $\mathrm{T} 3$ & 54 & $0.92(0.38-2.18, p=0.844)$ & $0.05(0.01-0.39, p=0.005)$ \\
\hline T4 & 20 & $3.41(1.42-8.23, p=0.006)$ & $0.08(0.01-1.05, p=0.055)$ \\
\hline \multicolumn{4}{|l|}{$\mathrm{pN}$} \\
\hline NO & 219 & - & - \\
\hline N1 & 157 & $1.58(0.89-2.82, p=0.117)$ & $1.13(0.32-3.92, p=0.850)$ \\
\hline $\mathrm{N} 2$ & 61 & $2.14(1.03-4.45, p=0.042)$ & $3.42(0.38-30.62, p=0.271)$ \\
\hline N3 & 24 & $2.20(0.75-6.44, p=0.149)$ & $0.36(0.04-3.52, p=0.382)$ \\
\hline \multicolumn{4}{|l|}{ PAM50 } \\
\hline Luminal A & 193 & - & - \\
\hline Luminal B & 99 & $1.89(1.02-3.51, p=0.044)$ & $3.09(0.97-9.85, p=0.057)$ \\
\hline Basal-like & 10 & $0.62(0.15-2.61, p=0.511)$ & $8.21(0.95-71.15, p=0.056)$ \\
\hline HER2-enriched & 10 & $3.83(1.14-12.84, p=0.030)$ & $20.69(3.20-133.85, p=0.001)$ \\
\hline Normal-like & 6 & $0.00(0.00-\operatorname{lnf}, p=0.997)$ & $0.00(0.00-\operatorname{Inf}, p=0.999)$ \\
\hline \multicolumn{4}{|l|}{ Methylation Cluster } \\
\hline Cluster 1 & 88 & - & - \\
\hline Cluster 2 & 133 & $1.55(0.75-3.19, p=0.239)$ & $0.89(0.28-2.88, p=0.852)$ \\
\hline Cluster 3 & 49 & $0.85(0.27-2.67, p=0.780)$ & $0.34(0.05-2.48, p=0.287)$ \\
\hline Cluster 4 & 168 & $1.70(0.84-3.44, p=0.142)$ & $0.87(0.29-2.65, p=0.813)$ \\
\hline Cluster 5 & 28 & $0.22(0.03-1.71, p=0.148)$ & $0.00(0.00-\operatorname{Inf}, p=0.997)$ \\
\hline \multicolumn{4}{|l|}{ CN Cluster } \\
\hline Cluster 1 & 59 & - & - \\
\hline Cluster 2 & 174 & $0.73(0.36-1.48, p=0.381)$ & $0.26(0.08-0.90, p=0.033)$ \\
\hline
\end{tabular}


Table 2 Univariate and multivariate analysis of the prognostic value of clinicopathological features in ER+/HER2- patients using Cox proportional hazard modeling in the TCGA dataset (Continued)

\begin{tabular}{lcll}
\hline Dependent & No. & HR (univariable) & HR (multivariable) \\
\hline Cluster 3 & 84 & $0.50(0.20-1.21, p=0.124)$ & $0.07(0.01-0.35, p=0.001)$ \\
Cluster 4 & 80 & $0.72(0.33-1.58, p=0.410)$ & $0.07(0.01-0.40, p=0.003)$ \\
Cluster 5 & 61 & $0.90(0.38-2.14, p=0.810)$ & $0.24(0.05-1.17, p=0.077)$ \\
miRNA Cluster & & & - \\
Cluster 1 & 33 & - & $0.22(0.03-1.56, p=0.130)$ \\
Cluster 2 & 78 & $0.62(0.22-1.73, p=0.363)$ & $0.03(0.00-0.72, p=0.030)$ \\
Cluster 3 & 22 & $0.40(0.08-1.92, p=0.250)$ & $0.39(0.10-1.63, p=0.200)$ \\
Cluster 4 & 120 & $0.63(0.26-1.53, p=0.308)$ & $1.96(0.33-11.82, p=0.461)$ \\
Cluster 5 & 40 & $1.00(0.36-2.77, p=1.000)$ & $0.58(0.11-3.06, p=0.524)$ \\
Cluster 6 & 92 & $0.64(0.26-1.56, p=0.330)$ & $0.38(0.04-3.66, p=0.403)$ \\
Cluster 7 & 39 & $0.55(0.17-1.74, p=0.308)$ & \\
\hline
\end{tabular}

Fig. 4. The insignificance of prognosis between the IGF-1 and EGFR defined groups could possibly be explained by the complexity of the PR loss mechanism which was contributed by a network of pathways.

\section{Discussion}

Although PR loss in ER+/HER2- breast cancer had a worse prognosis and showed insensitivity towards SERM therapy, the molecular mechanism related to PR loss remains to be equivocal. In this study, we analyzed ER+/ $\mathrm{PR}+$ and $\mathrm{ER}+/ \mathrm{PR}$ - breast cancer in the TCGA cohort. The PR-group was found to be more enriched in basallike mRNA subtype than the PR + group. Also, in terms of DNA methylation and copy number alteration features, the PR-group was also more similar to the basal-like subtype than the $\mathrm{PR}+$ group. Pathway activities which are calculated from transcriptomic and gene copy number data were comprehensively analyzed and compared between the two groups. The PR + and PR-groups could be classified using the pathway activities of 13 selected pathways. Growth factor pathways including EGFR and IGF-1 were found to be essential for the distinction of the two groups which agrees with previous studies. Although previous studies showed that both EGFR and IGF-1 pathway could suppress the PR expression in a way independent of ER [6, 19], our results found that while IGF-1 pathway activity was negatively correlated with the expression of PR, the EGFR pathway activity showed the opposite. Expression of PR was previously found to be repressed by activation of the IGF-1 pathway through PI3K/Akt/ mTOR signaling which was independent of ER [6]. Also, genes in PI3K/Akt/mTOR signaling pathways were upregulated in $\mathrm{ER}+/ \mathrm{PR}$ - breast cancer patients compared with $\mathrm{ER}+/ \mathrm{PR}+$ patients [20]. For the EGF pathway, studies were showing that activation of EGFR by adding EGF could enhance the phosphorylation of PR and promote its transcription activity [21], which could explain the positive correlation identified in our result between the EGFR pathway and PR expression. Other important pathways contributing to the classifying were ion-channel
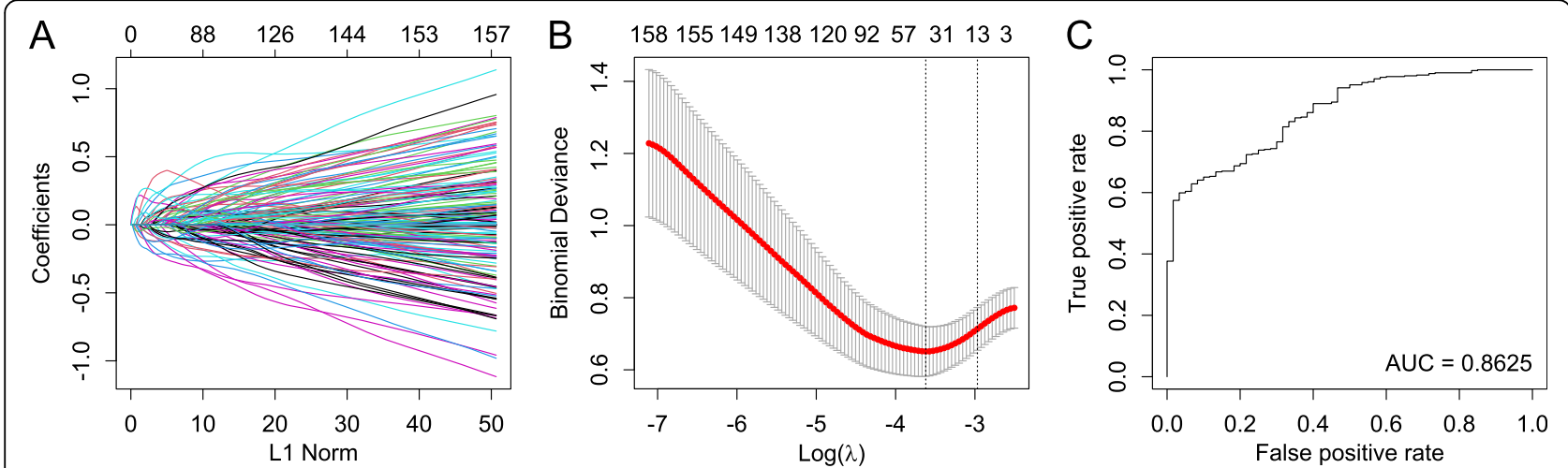

Fig. 3 Classifying ER+/PR + and ER+/PR- using 1387 pathways with LASSO methodology a Plots for LASSO regression coefficients over different values of the penalty parameter. $\mathbf{b}$ Cross-validation plot for the penalty term. $\mathbf{c}$ Receiver operating characteristic curve of the final model 
Table 3 Pathways selected in the final model by LASSO

\begin{tabular}{lr}
\hline Feature & Estimate \\
\hline (Intercept) & 1.27457 \\
Class_C/3_(Metabotropic_glutamate/pheromone_receptors) & -0.03500 \\
FOXA1_transcription_factor_network & 0.10619 \\
Signaling_by_Type_1_Insulin-like_Growth_Factor_1_ & 0.21581 \\
Receptor_(IGF1R) & \\
Amino_acid_transport_across_the_plasma_membrane & -0.01470 \\
Sema3A_PAK_dependent_Axon_repulsion & -0.07956 \\
Na_/Cl-_dependent_neurotransmitter_transporters & 0.02917 \\
Metal_ion_SLC_transporters & 0.05884 \\
IGF-1_signaling_pathway & 0.09220 \\
Signaling_by_constitutively_active_EGFR & -0.04876 \\
Reduction_of_cytosolic_Ca__levels & -0.13606 \\
NOTCH1_Intracellular_Domain_Regulates_Transcription & -0.04873 \\
Synthesis_of_Leukotrienes_(LT)_and_Eoxins_(EX) & 0.06633 \\
Activation_of_C3_and_C5 & 0.23174 \\
\hline
\end{tabular}

transporting pathway, immune-modulating pathway, and cell metabolism pathway. The above findings suggest that in ER+/HER2- breast cancers the various status of PR expression can be an indication of molecular variation, particularly for the growth factor pathway activation. Although, current clinical practice treats ER+/PR+/HER2and ER+/PR-/HER2- breast cancer patients in the same way, our study suggests that the PR-group should be more intensively studied to search for an effective therapy due to its poor prognosis. Our study, together with other researches, suggested that growth factor pathways such as IGF-1 pathway could be served as potential targets.

\section{Methods}

Data acquisition and pre-processing

UCSC Xena, an online exploration tool for public and private, multi-omic, and clinical/phenotype data, was used to download data of selected samples [22]. The 'TCGA Breast Cancer (BRCA)' cohort in the UCSC Xena was selected. All raw data used to generate Fig. 1; Table 1 was downloaded from the 'Phenotypes TCGA Hub', pathway scores were downloaded from the ' $z$ score of 1387 constituent PARADIGM pathways TCGA Hub'. The pathway scores were generated by the TCGA [23], using the PARADIGM-inferred activation of pathway features [24]. Surveillance, Epidemiology, and End Results (SEER) 18 registries research database (Nov 2018 Submission) was used for the analysis which includes cases diagnosed from 1975 to 2016 [25].

\section{Feature selection and LASSO regression}

Regularized regression is often applied in genetic studies of molecular phenotypes to select the most promising set of variants associated with a phenotype of interest [26]. A widely applied regularized regression method is LASSO, which adds a penalty term for the shrinkage of the parameter estimates to the least-squares loss function [27].

Feature selection and LASSO regression were performed using the "glmnet" $\mathrm{R}$ package [28]. The 'cv.glmnet' function was used to do the k-fold crossvalidation for glmnet, and the lambda.1se of the result was used as the optimal lambda. The glmnet function was used to fit a generalized linear model via penalized maximum likelihood. The alpha penalty was set to be 1 and the family was set to be 'binomial' in the glmnet function. The 'coef.glmnet' function was used to extract coefficients from the object generated by the glmnet
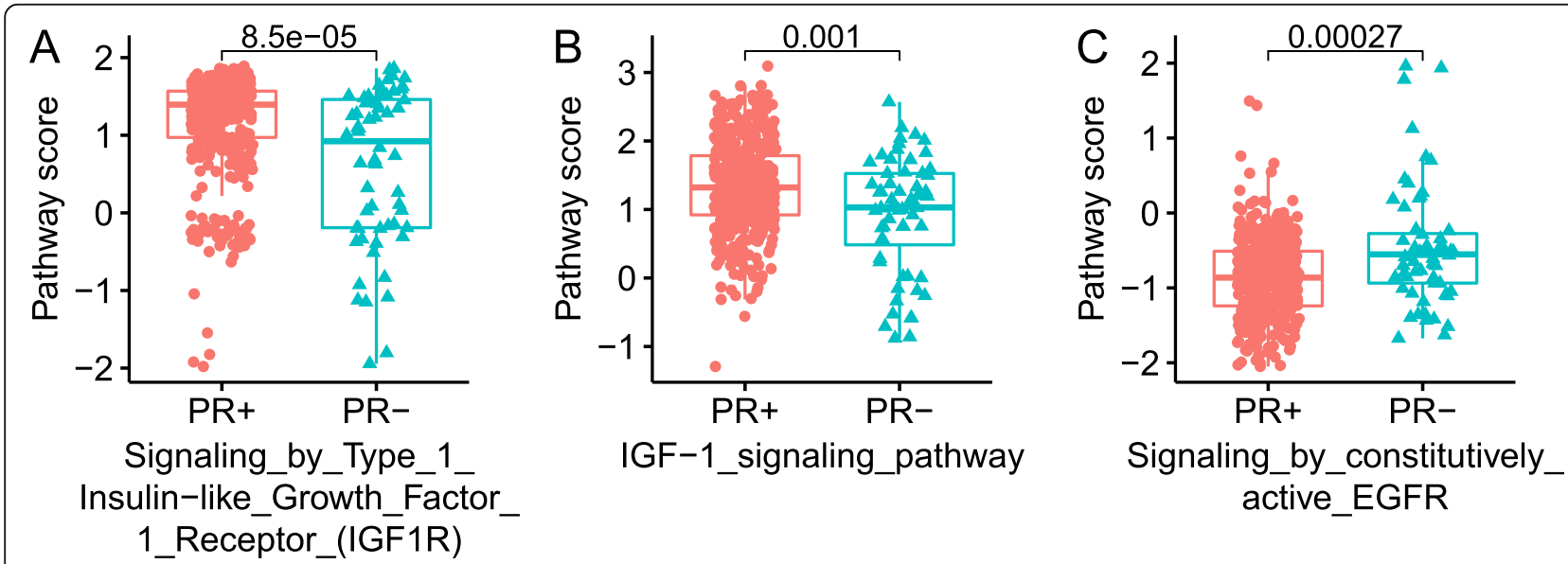

Fig. 4 Pathway scores of EGFR and IGF-1 pathways between the PR + and PR- groups. Box plot of the pathway scores in three selected pathways by LASSO between the two groups 


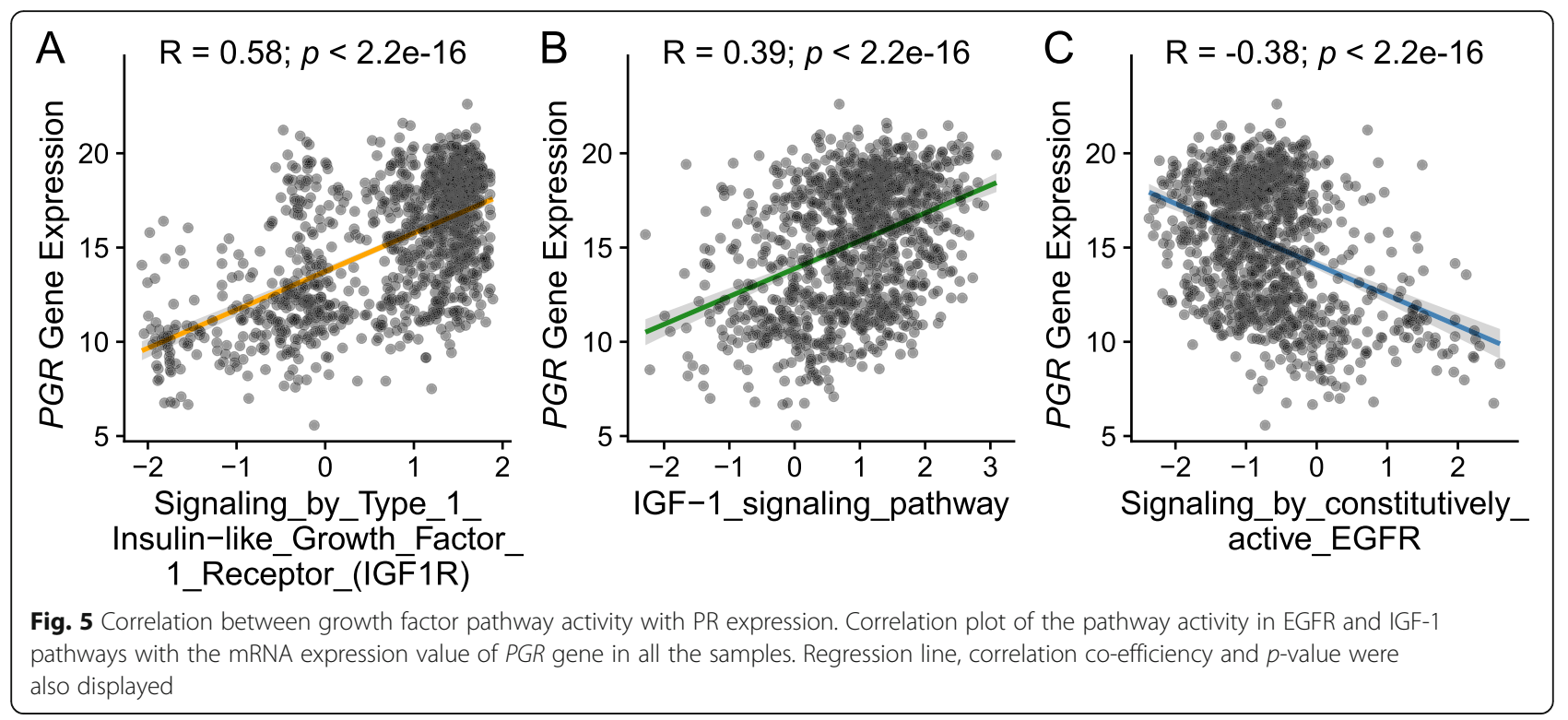

function, with the 's' argument being the optimal lambda generated by the cv.glmnet function.

\section{Statistical analysis}

Survival analyses were performed using the 'survival' (version 2.41) package [14]. The Kaplan-Meier method was used to estimate the survival outcomes of all patients by different categories; groups were compared using the log-rank statistic [29]. For univariate and multivariate analysis, the prognostic values of different clinicopathological factors were calculated by Cox proportional hazard modeling. The optimum threshold for distinguishing low and high activity groups was in similar method as described in our previous study [30]. $P$ values were calculated as two-sided, with statistical significance declared for $P$ less than 0.05 .

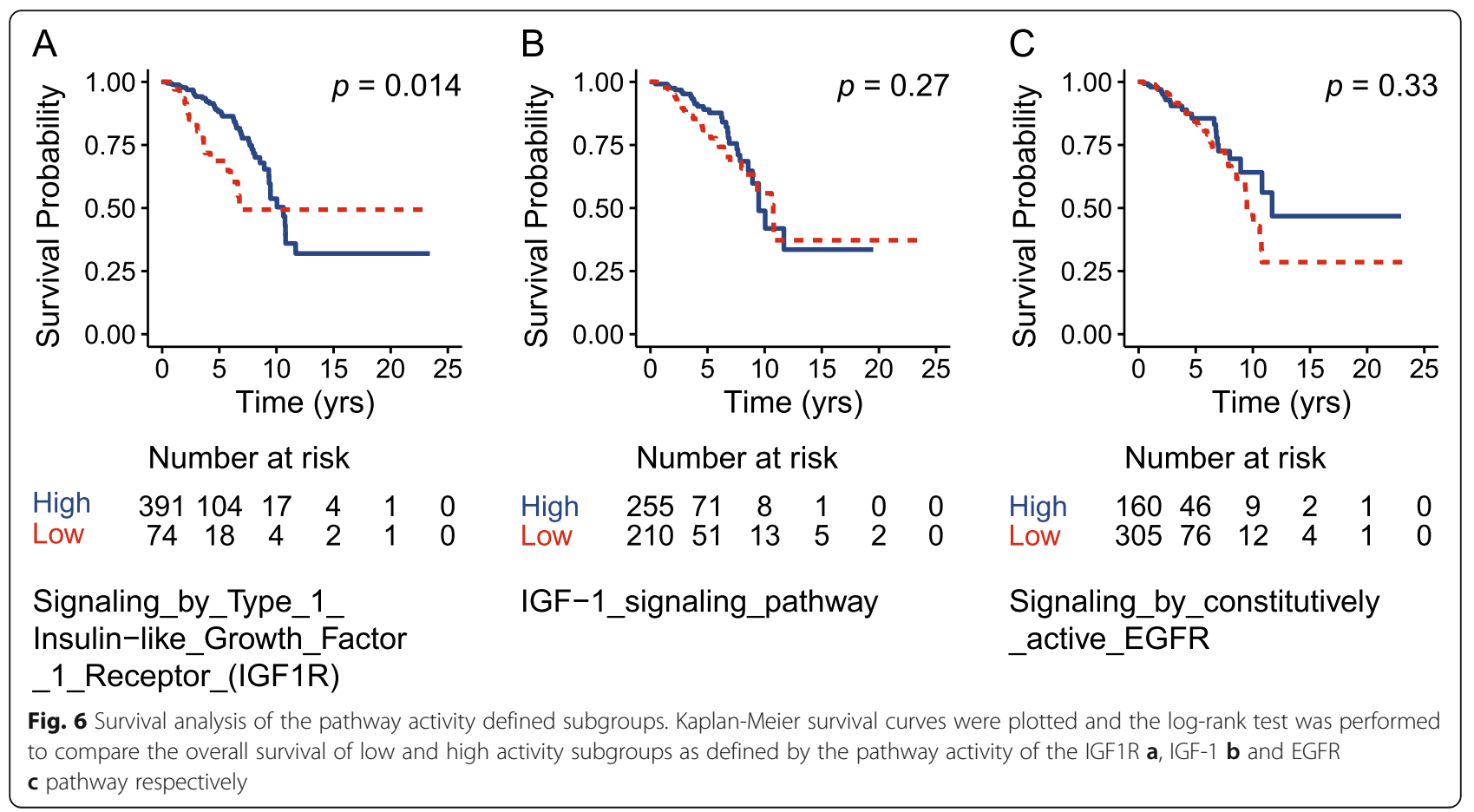




\section{Abbreviations}

AUC: Area Under the receiver operation characteristic Curve.; EGFR: Epidermal Growth Factor Receptor.; ER: Estrogen Receptor:; HER2: Human Epidermal growth factor Receptor 2.; IDC: Infiltrating Ductal Carcinoma.; IGF-1: Insulin-like Growth Factor-1.; ILC: Infiltrating Lobular Carcinoma.; LASSO: Least Absolute Shrinkage and Selection Operator.; NCD: National Cancer Database.; PR: progesterone receptor.; SEER: Surveillance, Epidemiology, and End Results.; SERM: Selective Estrogen Receptor Modulator.; TCGA: The Cancer Genome Atlas.

\section{Supplementary Information}

The online version contains supplementary material available at https://doi. org/10.1186/s12874-021-01297-8

Additional file 1. Table S1. Clinicopathological features of the two selected groups in the SEER dataset. Table S2. Univariate and multivariate analysis of the prognostic value of clinicopathological features in ER+/HER2- patients in the SEER dataset.

\section{Acknowledgements}

None.

\section{Authors' contributions}

TH and ML contributed to the conception of the study; TH, YC and JP performed the data analyses; $Y L$ and DZ contributed to the methodology; $Y L, D Z$ and JP helped the manuscript writing with constructive discussion; $M L$ wrote the final manuscript. The author(s) read and approved the final manuscript.

\section{Funding}

The author(s) disclosed receipt of the following financial support for the research, authorship, and/or publication of this article: This work was supported by the National Natural Science Foundation of China (Grant No. 82002979), Scientific Research and Development Funds of Peking University People's Hospital (Grant No. RDY2020-16) and Peking University Medicine Fund of Fostering Young Scholars' Scientific \& Technological Innovation supported by "the Fundamental Research Funds for the Central Universities" (Grant No. BMU2020PYB022, BMU2021PYB013).

\section{Availability of data and materials}

The dataset supporting the conclusions of this article is included within the manuscript.

\section{Declarations}

\section{Ethics approval and consent to participate}

As this study relied on publicly available data, no ethics approval was required.

\section{Consent for publication}

Not applicable.

\section{Competing interests}

None. All data used in this publication were generated by the TCGA project. The authors declare no competing interest.

\section{Author details}

'Department of Breast Disease, Peking University People's Hospital, Beijing, China. ${ }^{2}$ College of Life Sciences, University of Chinese Academy of Sciences, Beijing, China. ${ }^{3}$ Department of Pathology, Peking University Cancer Hospital, Beijing, China. ${ }^{4}$ Department of General Surgery, The Second Xiangya Hospital, Central South University, Changsha, Hunan, China. ${ }^{5}$ Department of Orthopedics, The Second Xiangya Hospital, Central South University, Changsha, Hunan, China
Received: 29 January 2021 Accepted: 23 April 2021

Published online: 22 May 2021

\section{References}

1. Ignatiadis M, Sotiriou C. Luminal breast cancer: from biology to treatment Nat Rev Clin Oncol. 2013;10(9):494.

2. Perou CM, Sørlie T, Eisen MB, Van De Rijn M, Jeffrey SS, Rees CA, Pollack JR, Ross DT, Johnsen H, Akslen LA. Molecular portraits of human breast tumours. Nature. 2000;406(6797):747-52

3. Jacobsen BM, Horwitz KB. Progesterone receptors, their isoforms and progesterone regulated transcription. Mol Cell Endocrinol. 2012;357(12):18-29.

4. Li Y, Yang D, Yin X, Zhang X, Huang J, Wu Y, Wang M, Yi Z, Li H, Li H. Clinicopathological characteristics and breast cancer-specific survival of patients with single hormone receptor-positive breast cancer. JAMA Netw Open. 2020;3(1):e1918160.

5. Vienonen A, Syvala H, Miettinen S, Tuohimaa P, Ylikomi T. Expression of progesterone receptor isoforms $A$ and $B$ is differentially regulated by estrogen in different breast cancer cell lines. J Steroid Biochem Mol Biol. 2002:80(3):307-13

6. Cui X, Zhang P, Deng W, Oesterreich S, Lu Y, Mills GB, Lee AV. Insulin-like growth factor-I inhibits progesterone receptor expression in breast cancer cells via the phosphatidylinositol 3-kinase/Akt/mammalian target of rapamycin pathway: progesterone receptor as a potential indicator of growth factor activity in breast cancer. Mol Endocrinol. 2003;17(4):575-88.

7. Yang C, Chen L, Li C, Lynch MC, Brisken C, Schmidt EV. Cyclin D1 enhances the response to estrogen and progesterone by regulating progesterone receptor expression. Mol Cell Biol. 2010;30(12):3111-25.

8. Dauphine C, Moazzez A, Neal JC, Chlebowski RT, Ozao-Choy J. Single Hormone Receptor-Positive Breast Cancers Have Distinct Characteristics and Survival. Ann Surg Oncol. 2020;27(12):4687-94.

9. Boland M, Ryan É, Dunne E, Aherne T, Bhatt N, Lowery A. Meta-analysis of the impact of progesterone receptor status on oncological outcomes in oestrogen receptor-positive breast cancer. Br J Surg. 2020;107(1):33-43.

10. Cui $X$, Schiff R, Arpino G, Osborne CK, Lee AV. Biology of progesterone receptor loss in breast cancer and its implications for endocrine therapy. J Clin Oncol. 2005;23(30):7721-35.

11. Daniel AR, Gaviglio AL, Knutson TP, Ostrander JH, D'Assoro AB, Ravindranathan P, Peng Y, Raj GV, Yee D, Lange CA. Progesterone receptor$B$ enhances estrogen responsiveness of breast cancer cells via scaffolding PELP1-and estrogen receptor-containing transcription complexes. Oncogene. 2015;34(4):506-15.

12. Balleine R, Earl M, Greenberg M, Clarke C. Absence of progesterone receptor associated with secondary breast cancer in postmenopausal women. $\mathrm{Br}$ 」 Cancer. 1999;79(9):1564-71.

13. Bamberger A-M, Milde-Langosch K, Schulte HM, Löning T. Progesterone receptor isoforms, PR-B and PR-A, in breast cancer: correlations with clinicopathologic tumor parameters and expression of AP-1 factors. Hormone Res Paediatr. 2000;54(1):32-7.

14. Liu X-Y, Ma D, Xu X-E, Jin X, Yu K-D, Jiang Y-Z, Shao Z-M. Genomic landscape and endocrine-resistant subgroup in estrogen receptor-positive, progesterone receptor-negative, and HER2-negative breast cancer. Theranostics. 2018;8(22):6386

15. Horowitz K, McGuire WL. Predicting response to endocrine therapy in human breast cancer: a hypothesis. Science. 1975;189(4204):726-7.

16. Mohammed H, Russell IA, Stark R, Rueda OM, Hickey TE, Tarulli GA, Serandour AA, Birrell SN, Bruna A, Saadi A. Progesterone receptor modulates ERa action in breast cancer. Nature. 2015;523(7560):313-7.

17. Allison KH, Hammond MEH, Dowsett M, McKernin SE, Carey LA, Fitzgibbons PL, Hayes DF, Lakhani SR, Chavez-MacGregor M, Perlmutter J. Estrogen and progesterone receptor testing in breast cancer: American society of clinical oncology/college of American pathologists guideline update. Arch Pathol Lab Med. 2020;144(5):545-63.

18. Network CGA. Comprehensive molecular portraits of human breast tumours. Nature. 2012:490(7418):61.

19. Thakkar JP, Mehta DG. A review of an unfavorable subset of breast cancer: estrogen receptor positive progesterone receptor negative. Oncologist 2011;16(3):276.

20. Creighton CJ, Osborne CK, van de Vijver MJ, Foekens JA, Klijn JG, Horlings HM, Nuyten D, Wang Y, Zhang Y, Chamness GC. Molecular profiles of 
progesterone receptor loss in human breast tumors. Breast Cancer Res Treat. 2009; 114(2):287-99.

21. Daniel AR, Qiu M, Faivre EJ, Ostrander JH, Skildum A, Lange CA. Linkage of progestin and epidermal growth factor signaling: phosphorylation of progesterone receptors mediates transcriptional hypersensitivity and increased ligand-independent breast cancer cell growth. Steroids. 2007: 72(2):188-201.

22. Goldman MJ, Craft B, Hastie M, Repečka K, McDade F, Kamath A, Banerjee A, Luo Y, Rogers D, Brooks AN: Visualizing and interpreting cancer genomics data via the Xena platform. Nat Biotechnol 2020:1-4.

23. Hoadley KA, Yau C, Hinoue T, Wolf DM, Lazar AJ, Drill E, Shen R, Taylor AM, Cherniack AD, Thorsson V. Cell-of-origin patterns dominate the molecular classification of 10,000 tumors from 33 types of cancer. Cell. 2018;173(2): 291-304 e296.

24. Vaske CJ, Benz SC, Sanborn JZ, Earl D, Szeto C, Zhu J, Haussler D, Stuart JM. Inference of patient-specific pathway activities from multi-dimensional cancer genomics data using PARADIGM. Bioinformatics. 2010;26(12):i237-45.

25. Howlader N, Noone AM, Krapcho M, Miller D, Brest A, Yu M, Ruhl J, Tatalovich Z, Mariotto A, Lewis D. SEER cancer statistics review, 1975-2016. Natl Cancer Institut. 2019. Online: https://seer.cancer.gov/csr.

26. Deutelmoser $H$, Scherer $D$, Brenner $H$, Waldenberger $M$, Suhre $K$, Kastenmüller G, Lorenzo Bermejo J. Robust Huber-LASSO for improved prediction of protein, metabolite and gene expression levels relying on individual genotype data. Brief Bioinform. 2020;bbaa230. https://doi.org/10.1 093/bib/bbaa230.

27. Tibshirani R. Regression shrinkage and selection via the lasso. Journal of the Royal Statistical Society: Series B (Methodological). 1996;58(1):267-88.

28. Friedman J, Hastie T, Tibshirani R. Regularization paths for generalized linear models via coordinate descent. J Stat Softw. 2010;33(1):1.

29. Bland JM, Altman DG. The logrank test. BMJ. 2004;328(7447):1073.

30. Long M, Hou W, Liu Y, Hu T. A Histone Acetylation Modulator Gene Signature for Classification and Prognosis of Breast Cancer. Curr Oncol. 2021; 28(1):928-39.

\section{Publisher's Note}

Springer Nature remains neutral with regard to jurisdictional claims in published maps and institutional affiliations.

Ready to submit your research? Choose BMC and benefit from:

- fast, convenient online submission

- thorough peer review by experienced researchers in your field

- rapid publication on acceptance

- support for research data, including large and complex data types

- gold Open Access which fosters wider collaboration and increased citations

- maximum visibility for your research: over $100 \mathrm{M}$ website views per year

At $\mathrm{BMC}$, research is always in progress.

Learn more biomedcentral.com/submissions 